\title{
Method for the Calculation of Orbits of Spectroscopic-Interferometric Binaries with Mixed Data
}

\author{
V. LANCHARES \\ Escuela Universitaria Politécnica, Universidad de Zaragoza, \\ Logroño, Spain
}

\begin{abstract}
From an initial value of the period and a fitted curve for the radial velocities an homotetic plane to that of the relative orbit is constructed from which $i, \Omega$ and parallax are deduced. The remaining orbital elements are obtained from the relations of the elliptical motion.
\end{abstract}

\section{INTRODUCTION}

We shall consider orbits of double stars for which we possess visual and radial velovity observations of the two components

$$
\begin{aligned}
& \left(t_{i}, v_{i 1}, v_{i 2}\right) ; \mathrm{i}=1, \ldots, \mathrm{N} \text { (spectroscopic obs.) } \\
& \left(t_{j}, \theta_{j}, \rho_{j}\right) ; \mathrm{j}=1, \ldots, \mathrm{M} \text { (visual obs.) }
\end{aligned}
$$

The period was determined using the method of B.W. Bopp et al. (1970) for both the spectroscopic and visual observations, suitably adapting the scheme.

Taking an arbitrary epoch $t_{0}$, the spectroscopic and visual observations are reduced to the interval $\left[t_{0}, t_{0}+P\right]$ or equivalently to $[0, P]$, and ordered acording to phase. For the set of spectroscopic observations $\left\{t_{i}, v_{i 1}-v_{i 2}\right\}$, a suitable approximation function $F(t)$ is constructed, with the condition

$$
\int_{0}^{P} F(t) d t=0 .
$$

The maximun and the minimun values of $F(t)\left(V_{M A X}, t_{M A X}\right)$ and $\left(V_{M I N}, t_{M I N}\right)$ correspond to the velocities at the ascending and descending nodes, respectively, were $z=0$.

\section{THE HOMOTETIC PLANE}

Let

$$
z(t)=\epsilon_{t_{M A X}}^{t} F(t) d t .
$$

To each observation $\left(t_{j}, \theta_{j}, \rho_{j}\right)$ we assign a value $z_{j}$. A plane homotetic to that of the relative orbit may be constructed, it being that which best fits the points of the form $\left(\rho_{j} \cos \theta_{j}, \rho_{j} \sin \theta_{j}, z_{j}\right)$. Let this plane $z=a x+b y+c$. It is evident that

$$
\tan \Omega=-\frac{a}{b}
$$

since a homotetic operation leaves the line of the nodes invariant. 
Moreover, $\left(V_{M A X}, t_{M A X}\right)$ and $\left(V_{M I N}, t_{M I N}\right)$ gives $K$ and $e \cos \omega$

$$
K=\frac{V_{M A X}-V_{M I N}}{2}, \quad e \cos \omega=\frac{V_{M A X}+V_{M I N}}{2 K}
$$

which in conjunction with the equation for the radial velocities

$$
v=K(e \cos \omega+\cos \phi) \approx F
$$

were $\phi=\omega+f$, allows $\cos \phi_{j}$ to be calculated for each visual epoch. Hence, with the equation

$$
\tan \phi_{j} \cos i=\tan \left(\theta_{j}-\Omega\right)
$$

a value for the inclination $i$ may be obtained.

The parallax is calculated from the ratio of the tangent of the inclination of the plane of the relative orbit to that of the inclination of the homotetic plane. This gives a factor for converting between seconds of arc and kilometers; hence, if the period is expressed in seconds $\alpha=\tan i / \tan I, I$ being the inclination of the homotetic plane. The parallax will be $\pi=a_{e} \alpha$, where $a_{e}$ is the semi-major axis of the terrestrial orbit.

\section{REMAINING ELEMENTS}

For the epochs $t_{M A X}$ and $t_{M I N}$, we obtain the corresponding values of $\rho$ by interpolation. Since we are considering the nodes these values correspond to the radii of the relative orbit:

$$
\rho=\frac{p}{1+e \cos \omega}, \quad \rho^{\prime}=\frac{p}{1-e \cos \omega}
$$

giving values for $e \cos \omega$ and for the parameter $p=a\left(1-e^{2}\right)$. Given that

$$
K=\frac{n a \sin i}{\sqrt{1-e^{2}}}, \quad \alpha K=\frac{n a \alpha \sin i}{\sqrt{1-e^{2}}}
$$

dividing $p$ by $\alpha K$ gives

$$
\frac{p}{\alpha K}=\frac{\left(1-e^{2}\right)^{3 / 2}}{n \sin i}
$$

from which may be deduced the eccentricity $e$ and, therefore, the semiaxis $a$ and the argument of the periastron $\omega$.

Lastly, to calculate the epoch of the periastron transit it is necessary to solve the equation

$$
F(t)=K \cos \omega(1+e) .
$$




\section{APPLICATION TO THE DOUBLE STAR 12 PERSEI}

From the data of A. Colacevich (1941) for the spectroscopic observations and H.A. McAlister (1977) for the visual observations, a period of 331 days can be deduced from either the spectroscopic or the visual observations. We take $t_{0}=$ 1899.696, this being the first epoch in which the radial velocity was observed, and order the observations with regard to phase. The approximation function $F$ for the radial velocities is a class $C_{2}$, piecewise, cubic polynomial, meeting the condition that the integral of $F$ is zero over one period. In the present paper we consider the following function

$$
F(t) \equiv\left\{\begin{array}{r}
-37.0799+4.49523 x-0.0748698 x^{2}+0.000356684 x^{3} \\
119.866-1.78259 x+0.0088344 x^{2}-0.0000153346 x^{3} \\
-30268.8+298.105 x-0.977639 x^{2}+0.00106632 x^{3}
\end{array}\right.
$$

in the intervals $[0,75],[74,304],[304,331]$. This function gives

$$
\begin{array}{ll}
V_{M A X}=46.1502 & t_{M A X}=43.6126 \\
V_{M I N}=-43.9744 & t_{M I N}=320.094
\end{array}
$$

with $k=45.0623$ and $e \cos \omega=0.0241421$.

The homotetic plane obtained is

$$
z=-85.5952+34191.1 x-29000.1 y
$$

giving $\Omega=229^{\circ} .696$ and $i=118^{\circ} .427$; the factor $\alpha=0.0000411539$ (the period in days). With the values of $\rho$ and $\rho^{\prime}$ obtained by interpolation we find that $e=0.698189, \omega=269^{\circ} .475$, and $a^{\prime \prime}=06595532$. Finally, $T=1899.99512(\mathrm{JD}$ 2415018.47).

\section{REFERENCES}

Bopp, B.W., Evans, D.S., Laing, J.D., \& Deeming, T.J. 1970, MNRAS, 147, 365

Colacevich, A. 1941, Oss. e Mem. Arcetri, 59, 16

McAlister, H.A. 1977, Proceedings of the 150th Meeting of American Astronomical Society 